\title{
Resolving the molecular fingerprint of the distal carboxy tail in modulating Cav1 calcium dependent inactivation
}

Lingjie Sang ${ }^{1}$, Daiana C. O. Vieira², David T. Yue ${ }^{1}$, Manu Ben-Johny ${ }^{1,3}$, Ivy E. Dick ${ }^{1,2}$

${ }^{1}$ Department of Biomedical Engineering, The Johns Hopkins University School of Medicine, Baltimore, MD.

${ }^{2}$ Department of Physiology, University of Maryland School of Medicine, Baltimore, MD.

${ }^{3}$ Department of Physiology and Cellular Biophysics, Columbia University, New York, NY.

Address correspondence to:

Ivy E. Dick

University of Maryland School of Medicine

Baltimore, MD 21210

ied@som.umaryand.edu

Running title: Defining the molecular interface between $\mathrm{Ca}_{\vee}$ IQ and ICDI

KeyWords: calcium channel; calmodulin (CaM); ion channel; calcium dependent inactivation (CDI); fluorescence resonance energy transfer (FRET); patch clamp 


\begin{abstract}
$\mathrm{Ca}^{2+} /$ calmodulin-dependent inactivation (CDI) of $\mathrm{Cav}$ channels is a critical regulatory process required for tuning the kinetics of $\mathrm{Ca}^{2+}$ entry for different cell types and physiologic responses. Calmodulin (CaM) resides on the IQ domain of the Cav carboxy-tail, such that $\mathrm{Ca}^{2+}$ binding initiates a reduction in channel open probability, manifesting as CDI. This regulatory process exerts a significant impact on $\mathrm{Ca}^{2+}$ entry and is tailored by alternative splicing. Cav1.3 and Cav1.4 feature a long-carboxy-tail splice variant that modulates CDI through a competitive mechanism. In these channels, the distal-carboxy-tail (DCT) harbors an inhibitor of CDI (ICDI) module that competitively displaces CaM from the IQ domain, thereby diminishing CDI. While this overall mechanism is now well-described, the detailed interaction loci for ICDI binding to the IQ domain is yet to be elucidated. Here, we perform alanine-scanning mutagenesis of the IQ and ICDI domains and evaluate the contribution of neighboring regions. We identify multiple critical residues within the IQ domain, ICDI and the nearby $A$ region of the channel, which are required for high affinity IQ/ICDI binding. Importantly, disruption of this interaction commensurately diminishes ICDI function, as seen by the re-emergence of CDI in mutant channels. Furthermore, analysis of the homologous ICDI region of Cav1.2 reveals a selective effect of this channel region on Cav1.3 channels, implicating a cross-channel modulatory scheme in cells expressing both channel subtypes. In all, these findings provide new insights into a molecular rheostat that fine tunes $\mathrm{Ca}^{2+}$ entry and supports normal neuronal and cardiac function.
\end{abstract}

\title{
Introduction
}

L-type voltage-gated calcium channels (Cav1.1-1.4) are an important conduit for extracellular $\mathrm{Ca}^{2+}$ entry into many excitable cells including cardiac myocytes, neurons, smooth muscle and skeletal muscle (1-4). As such, these channels are subject to rich and powerful modes of feedback regulation (5-7). In particular, $\mathrm{Ca}^{2+}$ dependent inactivation (CDI) of L-type channels is a crucial negative feedback mechanism that reshapes the electrical properties of neurons and cardiac myocytes and protects cells from $\mathrm{Ca}^{2+}$ overload (8-10). CDI is driven by the ubiquitous $\mathrm{Ca}^{2+}$ sensing molecule, calmodulin (CaM) $(10,11)$. Under basal $\mathrm{Ca}^{2+}$ conditions, $\mathrm{Ca}^{2+}$-free $\mathrm{CaM}$ (apoCaM) binds to the carboxy-terminal IQ domain of the channel and enhances channel openings (12). Upon elevation of intracellular $\mathrm{Ca}^{2+}$, the 'resident' CaM repositions on the channel, interacting with $\mathrm{Ca}^{2+} / \mathrm{CaM}$ binding sites located on the channel amino- and proximal carboxytermini $(13,14)$. This conformational change antagonizes the initial upregulation in channel open probability, which manifests as CDI. Not surprisingly, CDI of L-type channels has emerged as a key physiological process to limit excess $\mathrm{Ca}^{2+}$ influx during repetitive or sustained depolarization, and disruption of this feedback in the cardiac myocytes may lead to lethal cardiac arrhythmias $(15,16)$. This stereotypic behavior, however, diverges in multiple physiological settings where strong CDI of L-type channels is curtailed, thus permitting $\mathrm{Ca}^{2+}$ channels to faithfully respond to a tonic stimulus. For example, in photoreceptors and bipolar cells, endogenous Cav1.4 exhibit minimal $\mathrm{CDI}$, thereby allowing sustained $\mathrm{Ca}^{2+}$ influx and slow, graded changes in the membrane potential necessary for tonic glutamate release and normal vision $(17,18)$. Similarly, Cav1.3 channels in the inner hair cells also lack CDI (19). Beyond these, the basal strength of Cav1.2 and Cav1.3 CDI vary in different neuronal subtypes in the central nervous system, suggesting a sophisticated scheme of $\mathrm{Ca}$ v channel feedback ripe with physiological insights (20). 
The molecular mechanisms that fine-tune L-type channel CDI are two-fold and have been of longstanding interest. One scheme involves channel-interacting proteins such as calmodulin-like $\mathrm{Ca}^{2+}$-binding proteins (CaBP1-4) $(18,19,21,22)$ and $\mathrm{SH} 3$ and cysteine rich domain containing proteins (stac1-3) (23-26) that suppress CDI utilizing an allosteric or mixed-allosteric mechanism. In contrast, Cav1.3 and Cav1.4 channels may intrinsically disable CDI via an alternatively-spliced specialized CDI-inhibiting module (ICDI) within the distal carboxy-tail of the channel (20,27-33). The latter form of regulation is complex and bears important biological consequences. First, splice-inclusion of the distal carboxy-tail occurs in a cell-type dependent manner. For instance, alternative splicing of Cav1.3 results in variable inclusion of the ICDI domain in distinct regions of the brain and in the sinoatrial node, enabling precise tuning of CDI in these cell types $(27,31,34,35)$. Second, Cav1.2 appears to harbor a highly homologous ICDI region (33), yet CDI for this channel is known to be robust, both when evaluated as full length channels in heterologous expression system, as well as in primary cells where the C-tail containing ICDI is believed to be cleaved off the channel $(9-11,33)$. As such, the function of the ICDI module within Cav1.2 channels remains unclear. Third, the inhibition of CDI by ICDI is the result of competitive binding by apoCaM versus ICDI with the channel IQ domain $(20,31)$. In addition to diminishing CDI, the displacement of apoCaM results in a dramatic decrease in baseline channel open probability (12). Fourth, adding to the richness the modulatory role of ICDI, RNA editing and/or fluctuations in cytosolic CaM concentrations can tune the extent of this competition, enabling different degrees of CDI tailored to specific cell types or physiologic states $(12,36)$. Importantly, pathologic changes to this system may be linked to altered CaM concentrations in Parkinson's disease and heart failure $(37,38)$, and mutations within the ICDI of Cav1.4 channels are known to be causative of congenital stationary night blindness $(32,39,40)$. Thus, the modulation of CDI by ICDI stands as a critical and robust mechanism for adapting channel regulation to select cell types and conditions. Moreover, as the number of known pathogenic mutations within LTCCs continues to grow, the ability to map these mutations to a locus with known mechanistic impact would enable rapid insight into the pathogenesis of LTCC channelopathies.

Although the overall competitive nature of ICDI regulation of L-type channels is now wellestablished (20,31), the precise binding interfaces involved in this regulation are yet to be identified. This gap in understanding is critical as mutations in the carboxy-tail of LTCCs result in neurological disease $(32,39,40)$. Furthermore, a residue-level analysis may shed light upon structural differences between the ICDI domain of Cav1.3 and the homologous segment of Cav1.2 that engender differential functional regulation. To characterize the landscape of the IQ/ICDI interaction of L-type channels, here we undertook systematic alanine scanning mutagenesis of both IQ and ICDI domains. Through live-cell FRET two-hybrid binding assays and electrophysiological analysis, we identified several novel hotspots on both IQ and ICDI segments that mediate a high-affinity interaction and are functionally relevant for CDI inhibition. Systematic analysis of these mutations revealed a strong inverse correlation between the strength of CDI and the binding affinity of the ICDI domain for the IQ segment, as predicted for a competitive inhibitor $(13,14,20)$. Thus, we have identified residues which alter binding in a functionally relevant manner. Moreover, similar critical residues were identified in adjacent regions, defining a comprehensive interface map of the IQ/ICDI interaction. Finally, extending our analysis to Cav1.2 channels, we found that the ICDI module binds to the Cav1.2 IQ domain with a reduced affinity, 
and that this binding is insufficient to cause more than a nominal change in the CDI of full length channels. However, the ICDI from Cav1.2 is capable of binding the Cav1.3 IQ region with high affinity, resulting in a much larger decrease in CDI of these channels. Given the propensity of the C-tail of Cav1.2 to exist as a separate peptide within myocytes and neurons $(27,33,41,42)$, these findings raise the prospect of a cross-channel feedback scheme in some cell types. Overall, these results elucidate the detailed binding interface between components of the carboxy-tail of L-type $\mathrm{Ca}^{2+}$ channels, lending new insight into normal and pathologic channel regulation.

\section{Results}

\section{Identification of critical residues within the IQ domain necessary for ICDI}

To identify key residues that support a high-affinity IQ/ICDI interaction, we undertook systematic alanine substitution of the IQ domain and evaluated both the relative binding affinity and the strength of ICDI mediated inhibition of CaM regulation. Importantly, the ICDI domains of both Cav1.3 and Cav1.4 are highly homologous, and have been shown to interact with IQ domains in like manner evoking similar functional effects $(20,27,29)$. Even so, the ICDI domain from Cav1.4 $\left(\mathrm{ICDI}_{1.4}\right)$ has a greater binding affinity for the IQ domains of both Cav1.3 and Cav1.4, with FRET binding assays yielding more robust measurements with enhanced signal to noise ratio as compared to ICDI $_{1.3}$ (43). We therefore focus on this canonical ICDI motif for our studies. However, robust expression of the holo-Cav1.4 channel in recombinant systems is notoriously challenging, largely due to their diminutive open probability $(12,44)$. We therefore chose to explore the interaction between the IQ domain of Cav1.3 channels $\left(I_{1.3}\right)$, and ICDI $\left.\right|_{1.4}$. To this end, we utilized a chimeric channel in which the DCT of Cav1.4 is spliced onto the backbone of Cav1.3 (Cav1.3 ${ }_{\triangle / \text { DCT1.4) }}$ (Fig. 1A), which has previously proven useful in dissecting the mechanisms underlying ICDI modulation of the channel $(12,20)$. This chimera furnishes a strong IQ/ICDI interaction coupled with a robust functional readout, enabling quantitative analysis.

To begin, we confirm the functional impact of ICDI in our chimeric channel by evaluating the extent of CDI in HEK293 cells. Indeed, CDI is entirely abolished in Cav1.3 ${ }_{\triangle 1626 / D C T 1.4}$, as seen by the identical $\mathrm{Ba}^{2+}$ and $\mathrm{Ca}^{2+}$ current decay in response to a depolarizing pulse (Fig. 1B). However, removal of $\mathrm{DCT}_{1.4}$ restores robust $\mathrm{CDI}$, as seen by the rapid decay of the $\mathrm{Ca}^{2+}$ current (Fig. 1C, red). In contrast, when $\mathrm{Ba}^{2+}$ (which binds poorly to $\mathrm{CaM}$ ) is used as the charge carrier, there is minimal inactivation (Fig. 1C, black). We therefore define the extent of CDI as the ratio of $\mathrm{Ca}^{2+}$ current remaining after $300 \mathrm{~ms}$ of depolarization versus $\mathrm{Ba}^{2+}$ current at the same time point.

We next utilized a FRET 2-hybrid binding assay $(45,46)$ to evaluate the relative strength of interactions between the IQ and ICDI regions. FRET binding pairs were constructed by tagging cerulean fluorescent protein to ICDI ${ }_{1.4}$, and venus fluorescent protein to PrelQ ${ }_{3}-I_{Q}-A_{1.3}$, a peptide that includes $I Q_{1.3}$ as well as $\sim 30$ residues upstream $\left(P_{3} I_{2}\right)$ and $\sim 150$ residues downstream (Aregion) of the IQ domain (Fig. 1D, Fig. S1). Both $\mathrm{Prel}_{3}$ and $A$ regions were included initially to ensure that all likely interacting residues were included. Strong binding was detected between the Venus-PrelQ $\mathrm{Q}_{3}-\mathrm{IQ}_{\mathrm{A}} \mathrm{A}_{1.3}$ and Cerulean-ICDI ${ }_{1.4}$, as can be appreciated by the steep FRET binding curve determined by the FRET Ratio (FR) of each cell plotted as a function of the free donor concentration (Cerulean tagged $\mathrm{ICDI}_{1.4}$ ) (Fig. 1D, black). After calibration, the FRET binding curve for WT Venus-PrelQ ${ }_{3}-I_{Q}-A_{1.3}$ versus Cerulean-ICDI 1.4 yielded a $K_{a}$ of $21.4 \mu \mathrm{M}^{-1}$. To identify key 
residues that support a high-affinity IQ/ICDI interaction, we undertook systematic alanine substitution of the IQ domain and evaluated the effect on binding affinity in our FRET assay. Within $I Q_{1.3}$, we substituted each residue with an alanine or, at loci where the wild-type channel featured an alanine, we replaced the residue with a threonine. For identification of each residue, the canonical isoleucine is assigned position 0 . Application of our FRET assay to each mutated peptide identified three residues, Y[-5]A, F[-2]A and F[+4]A, which severely perturbed the IQ/ICDI interaction (Fig. 1E, Fig. S1). Focusing on F[-2]A, FRET binding produced a shallower curve as compared to WT (Fig. 1D blue vs. gray), resulting in a $K_{\mathrm{a}}$ of $5.8 \mu \mathrm{M}^{-1}$ (Fig. 1E, blue). Introducing this mutation into the chimeric channel resulted in a partial rescue of CDI (Fig. 1F), indicating that this interaction site is functionally relevant. However, the IQ domain substitutions $Y[-5] A$ and $\mathrm{F}[+4] \mathrm{A}$, which also had a marked effect on $K_{\mathrm{a}}$, resulted in minimal CDI rescue (Fig. 1F, G, Fig. S2). Importantly, these residues also serve as anchors for apoCaM binding to the Cav1.3 IQ domain, resulting in weak baseline CDI even in the absence of the ICDI domain (Table S1) (13).

In order to confirm the functional relevance of each binding loci identified, we turned to a previously described analysis known as individually transformed Langmuir (iTL) analysis (14). This approach allows us to rigorously correlate relative changes in binding with functional changes in CDI. As iTL was initially derived to evaluate the binding interfaces critical to CaM mediated channel regulation $(13,14)$, we adjust the model to reflect the competitive binding scheme between CaM and ICDI (20). To do so, we first account for the ambiguity caused by binding sites which are important for both apoCaM and ICDI binding. We therefore incorporate both the IQ domain's intrinsic affinity for apoCaM $\left(K_{\mathrm{a}-\mathrm{CaM}}\right)$ and that for the ICDI segment $\left(K_{\mathrm{a}-\mathrm{ICDI}}\right)$, the competitive inhibitor, by adjusting our measured $K_{\mathrm{a}-\mathrm{ICDI}}$ such that:

$$
K_{a-I C D I}^{c o r r}=\frac{K_{a-C a M}^{W T}}{K_{a-C a M}^{\text {mut }}} \cdot K_{a-I C D I}
$$

Where $K_{a-C a M}^{W T}$ is the apoCaM binding affinity of WT PrelQ $-I Q-\mathrm{A}_{1.3}$, and $K_{a-C a M}^{\text {mut }}$ represents the apoCaM binding affinity of each mutant peptide, values which were previously measured (13) and are listed in Table S1. This compensation remains valid provided that the local concentration of ICDI is much greater than $K_{\mathrm{d}-I C D I}\left([I C D I]>>1 / K_{\mathrm{a}-I C D I}\right)$. With this adjustment made, CDI can be defined by a modified Langmuir function as follows:

$$
\frac{C D I}{C D I_{\max }}=\frac{[\text { apoCaM }] \cdot K_{a-C a M}}{1+[\text { apoCaM }] \cdot K_{a-C a M}+[I C D I] \cdot K_{a-I C D I}^{c o r r}}
$$

where $C D I$ is the strength of $C D I$ under endogenous levels of CaM; $C D I_{\text {max }}$ is the $C D I$ in saturating concentrations of CaM (Table S1); $K_{\mathrm{a}-\mathrm{CaM}}$ is the association constant for apoCaM binding to PrelQ $_{3}-I_{Q}-A_{1.3}$; [apoCaM] is the free apoCaM concentration in the cell; and [ICDI] is the effective local concentration of ICDI. Equation 2 predicts an inverse correlation between $K_{a-I C D I}^{c o r r}$ and relative $C D I$, which we can fit to our data $(\mathbf{F i g} .1 \mathrm{H})$. For channels containing $W T \mathrm{IQ}_{1.3}$ and $\mathrm{ICDI}_{1.4}$ domains, the strong binding between IQ and ICDI results in minimal CDI, as seen by the black data point on the plot. In contrast, Cav1.3s channels, which lack an ICDI domain such that $K_{a-I C D I}^{c o r r}$ $=0$ by definition, display large CDI values (cyan). The F[-2]A mutation, which produced a partial 
restoration of CDI, resides in an intermediate position (blue). Overall, our data can be well fit by equation 2 (Fig. 1H), confirming the functional relevance of the identified residues in a competitive model.

\section{Alanine scanning of the ICDI domain reveals complementary hotspots}

Having identified several critical residues within the IQ domain required for ICDI binding, we next probed the ICDI for critical determinates of binding to the IQ. In order to scan a more extensive segment of the channel, we made triple alanine substitutions for every three contiguous residues within the ICDI domain. The ICDI domain has previously been localized to amino acids 18681956 of the Cav1.3 DCT $(29,32,33)$. We therefore undertook our alanine scan on this segment of the channel. Importantly, this region includes the distal C-terminal regulatory domain (DCRD), which was previously identified as playing an important role in the ICDI mediated inhibition of CDI $(27,31,33,47)$. Finally, we included a selective V[1907]A mutation, as this amino acid change has previously been shown to dramatically alter the function of ICDI (30). To evaluate the effect of these alanine substitutions on IQ/ICDI binding, we again utilized our FRET two-hybrid binding assay, pairing Venus-PrelQ ${ }_{3}-I Q_{2}-A_{1.3}$ with Cerulean-ICDI ${ }_{1.4}$ (Fig. 2A, Fig. S3). Indeed, measured $K_{\mathrm{a}}$ values revealed multiple hotspots within the ICDI domain, with a wide range of binding affinities with the IQ containing peptide (Fig. 2B). The two mutation sites displayed in gray (KQE[1911]AAA and YSD[1941]AAA) were not evaluated as they failed to express. Notably, the effects of the hotspots on the ICDI domain were significantly larger than those observed within the IQ domain (Fig. 2B vs. Fig. 1E).

In order to correlate loss of binding affinity with function, we measured the CDI those mutations which exhibited a large change in binding affinity (Fig. 2C, Fig. S4). As predicted, mutations which resulted in a significant loss of IQ/ICDI binding also exhibited a corresponding restoration of CDI. Focusing on two examples, IAD[1914]AAA moderately reduced IQ/ICDI binding (Fig. 2A,B, red), while introduction of the same mutations into our chimeric channel enabled a partial restoration of CDI (Fig. 2C, D, red). On the other hand, SLV[1886]AAA displayed a drastic reduction in IQ/ICDI binding (Fig. 2A, B green), and CDI was fully restored to the level seen in Cav1.3 ${ }_{\Delta 1626}$ (Fig. 2C, D green). Importantly, all identified loci are well fit by our Langmuir function, such that the same set of equation 2 parameters describes both the IQ region and ICDI (Fig. 2E). Of note, as mutations in ICDI do not affect the binding of apoCaM, the correction factor for $K_{a}$ is no longer required, and $K_{a-I C D I}^{c o r r}=K_{a-I C D I}$. Having identified critical loci within ICDI we note that one of these amino acids (S[1866]) has previously been identified as a phosphorylation site which reduces the binding affinity of $\mathrm{ICDI}_{1.4}$ by about 10 fold, while increasing CDI of Cav1.3 ${ }_{\triangle 1626 / D C T 1.4}$ (43). We therefore included the results of phosphorylation at this site in our analysis (Fig. 2E, orange). Indeed, phosphorylation of this amino acid results in a change in binding affinity which correlates with CDI according to the same Langmuir function. Thus, we have identified numerous residues within $\mathrm{ICDI}_{1.4}$ which are critical determinants of a functional competition between ICDI and apoCaM for the IQ region of Cav1.3.

\section{The role of the A region in the IQ/ICDI interaction}

While our analysis identified several functionally-relevant IQ domain loci, the impact of these mutations was far less than those identified within the ICDI (Fig. 1 vs. Fig. 2). This suggests that additional regions outside the IQ domain may contribute to ICDI binding. In order to identify such 
regions, we generated truncated variations of our Venus-PreIQ $-I Q-A_{1.3}$ construct and paired them with Cerulean-ICDI ${ }_{1.4}$ in the FRET two-hybrid binding assays (Fig. 3A, Fig. S5). We began by removing the PrelQ $\mathrm{Q}_{3}$, and found no change in FRET binding, indicating that all relevant interaction loci are contained within the IQ and A regions (Fig. 3B, blue). However, removal of the IQ domain, leaving only the A region intact, resulted in a complete loss of FRET binding (Fig. 3B, open circles). Likewise, the IQ region alone displayed no binding with ICDI (Fig. 3C, B), suggesting that both the IQ and the A region are necessary for interaction with ICDI. In order to further localize the critical interaction sites, we undertook successive truncation of the vernus-IQ- $A_{1.3}$ peptide (Fig. 3D). FRET measurements demonstrated minimal effect of truncations up to 34 amino acids from the end of the A region, as demonstrated by the strong binding of Venus-IQ- $A_{1.3} \Delta 34$ with ICDI $I_{1.4}$ (Fig. 3E, green). However, our next truncation, venus-IQ- $A_{1.3} \Delta 28$, exhibited a marked decrease in FRET binding (Fig. 3E, F, red) Thus, the 34 residues immediately downstream of the IQ domain critically augment ICDI binding. Of note, this region includes the previously identified proximal Cterminal regulatory domain (PCRD), which is reported to play an important role in the ICDI interaction $(27,31,33,47)$. Having identified a subset of the A region which is vital to ICDI binding, we again undertook systematic alanine substitutions, replacing each 3 contiguous residues with three alanine residues and undertook our FRET-2-hybrid binding assay (Fig. 3G). Disruptions in binding were identified as the result of a number of mutations, spanning both the previously identified PCRD region as well as a previously unidentified region upstream of this motif $(\mathbf{F i g}$. $\mathbf{3 H}$, I, Fig. S6). Thus, both the $I Q$ and the distal A region of the channel are required for high-affinity interaction with ICDI.

\section{The functional relevance of ICDI in Cav1.2 channels}

Similar to Cav1.3 and Cav1.4, Cav1.2 channels also feature a highly homologous ICDI segment, argued to function as a channel inhibitor $(33,48)$ or as a transcriptional factor $(41,42)$. We therefore considered the impact of $\mathrm{ICDI}_{1.2}$ on both $\mathrm{Ca}_{\mathrm{v}} 1.2$ and $\mathrm{Ca}_{\mathrm{V}} 1.3$ channels. We interrogated the binding of Cerulean-ICDI ${ }_{1.2}$ with Venus-PrelQ $3-I_{2}-A_{1.2}$ via FRET two-hybrid (Fig. 4A, B), and found that the interaction is significantly weaker than the prototypic Venus-PrelQ $Q_{3}-I Q-A_{1.2}$ and Cerulean-ICDI ${ }_{1.4}$ interaction (Fig. 4B vs. Fig. 1D). However, when paired with Venus-PrelQ $Q_{3} I Q_{-}$ $\mathrm{A}_{1.3}$, binding with $\mathrm{ICDI}_{1.2}$ is significantly larger, and only about half that of the strong binding of $I_{1 C D I}$ (Fig. 4C). Thus, it appears that $\left.I_{1 C D I}\right|_{1.2}$ is poised to have a larger effect in the context of Cav1.3 channels as compared to its native channel backbone. Nonetheless, the limited binding observed between Cerulean-ICDI ${ }_{1.2}$ with Venus-PrelQ $\mathrm{Q}_{3}-\mathrm{IQ}_{2}-\mathrm{A}_{1.2}$ prompted us to evaluate the possibility of a functional role for ICDI 1.2 within Cav1.2 channels. Interestingly, truncation of Cav1.2 at the known carboxy-tail cleavage site (49) for this channel (Cav1.2 $\left.{ }_{\Delta 11800}\right)$ resulted in a minimal, yet statistically significant ( $p \leq 0.05$ ), increase in CDI (Fig. 4D, F, Fig. S7). Next, to test the effect of ICDI ${ }_{1.2}$ on Cav1.3 channels we replaced the native ICDI ${ }_{1.3}$ of Cav1.3 long channels with ICDI ${ }_{1.2}$. Indeed, the loss of CDI surpassed that of Cav1.2 channels (Fig. 4E), as predicted based on the stronger PrelQ $\mathrm{Q}_{3}-\mathrm{IQ}-\mathrm{A}_{1.3} / \mathrm{ICDI}_{1.2}$ interaction (Fig. 4C). In fact, the CDI exhibited by Cav1.3-ICDI 1.2 channels was not statistically different than the CDI measured in the native Cav1.3 long splice variant (Fig. 4F).

Multiple studies have shown that the DCT of Cav1.2, containing ICDI $\left.\right|_{1.2}$, exists as a peptide within neurons and cardiomyocytes, either due to proteolysis $(33,49)$, or as a result of alternative 
transcriptional initiation sites (41). Moreover, it has previously been demonstrated that ICDI domains can exert their effects on L-type channels when expressed as separate peptides (27). We therefore sought to recreate the potential interaction of select Cav1.3 channel variants with the DCT of Cav1.2. To begin, we choose the human Cav1.343s splice variant of Cav1.3, as these channels terminate just past the A region and thus lack an inherent ICDI module (27). In addition, the inclusion of the $A$ region within $\mathrm{Ca} 1.3_{435}$ has been demonstrated to be important for ICDI binding, both in functional experiments done by others (27) and in our alanine scan of the A region (Fig. 3). We therefore generated the proteolytic product of human Cav1.2 channels (DCT 1.2 ), and evaluated the effect of this peptide on the CDI of Cav1.343s. Indeed, consistent with previous studies (27), co-expression of $\mathrm{DCT}_{1.2}$ significantly reduced the CDI of Cav1.343s (Fig. 4G, Fig. S7). For comparison, we also co-expressed these channels with ICDI ${ }_{1.4}$ expressed as a peptide, which we have shown has a $K_{\mathrm{a}}$ about double that of $\mathrm{ICDI}_{1.2}$ (Fig. 4C vs. Fig. 1D). Indeed, ICDI ${ }_{1.4}$ results in an even larger CDI deficit when expressed with Cav1.343s (Fig. 4G). Thus, the proteolytically cleaved $\mathrm{DCT}_{1.2}$ is well poised to exert a significant modulation of select Cav1.3 channel variants, such that the ambient concentration of $\mathrm{CaM}_{\text {and }} \mathrm{DCT}_{1.2}$ are able to tune the CDI of Cav1.343s channels in a competitive manner (Fig. 4H).

\section{Discussion}

CaM regulation of Cav channels is vital to normal physiology, and thus has been the subject of intense study $(8,10,11,17,50-52)$. The competitive mechanism of ICDI within select Cav1 channels forms a basis with which CaM regulation can be tuned $(12,20,32)$. Numerous processes designed to modulate this regulation include splice variation, RNA editing, variations in ambient CaM concentration and phosphorylation $(12,13,18,36,43)$. Identification of critical loci involved in this regulation is therefore key to understanding how CaM regulation may vary in different physiological and pathological states. As such, in-depth residue-level analysis not only reveals interfaces utilized by cells to tune channel regulation, but may offer targets in the search for novel regulators of the channel which may have therapeutic benefit. In particular, the dramatically different efficacy of ICDI across channel subtypes may offer the possibility of subtype selective drug targeting, which remains challenging for Cav1 channels.

Given the importance of understanding these interactions within the C-tail of Cav1 channels, we quantified the structure-function relationship of these interactions using a variant of previously described iTL analysis (14). This provided a major advantage in that the quantitative agreement of our results with equation 2 demonstrates that each identified locus is functionally relevant. This overcomes a common limitation of binding assays between channel fragments, which may identify sites which are inaccessible or inconsequential in the context of the holochannel. Moreover, by fitting to a specific Langmuir curve, we can distinguish mutations which may alter channel function through ancillary mechanisms such as transduction or altered folding of the channel. Thus, in addition to identifying critical loci, our results confirm the competitive mechanism described for ICDI modulation of CDI.

A number of previous studies have identified regions within the $\mathrm{C}$-tail of $\mathrm{Cav} 1$ channels which are critical to the competitive mechanism of ICDI inhibition. Among these are the PCRD and DCRD regions, which were identified within Cav1.2 channels as potential interaction sites such that the 
DCRD region of the proteolytically cleaved Cav1.2 DCT may interact with the PCRD on the channel via electrostatic interaction with the negatively charged amino acids (33). Homologous regions within Cav1.3 and Cav1.4 were later shown to be important for ICDI regulation, and for the interaction with modular peptides $(27,30,31,33,47)$. In this study, the PCRD resides within the A region, and overlaps with the identified locus of critical amino acids required for high affinity binding between the IQ-A and ICDI. Interestingly, these critical amino acids were identified both within the PCRD, as well as upstream of the motif, arguing for a larger interacting region which is highly conserved across Cav channels (Fig. S8). However, our binding assay also demonstrated that the A region, in itself, is insufficient for high affinity binding, but also requires the upstream IQ region (Fig. 3A). This fits with previous findings in which neutralization of the PCRD arginines was not sufficient to prevent the ICDI inhibition of CDI (31), pointing to the existence of additional interaction loci. In a similar manner, our scan of the ICDI region validated the importance of the DCRD, while also identifying numerous critical interacting loci upstream of the motif (Fig. S8). Thus, this study has expanded our knowledge of the important interactions required for ICDI inhibition, providing a comprehensive map of the critical loci within the C-tail. The impact of ICDI in Cav1.3 and Cav1.4 channels has been well recognized, however its role in Cav1.2 has been uncertain. It had been demonstrated that the truncation of Cav1.2 results in increased current density, altered voltage dependence of channel activation and disrupted targeting of the channel to the membrane $(33,53,54)$, however no impact on CDI has been reported. Here, we find that the impact of $\mathrm{ICDI}_{1.2}$ within Cav1.2 channels is minimal, allowing cleavage of this DCT region without significant disruption of CDI. Yet ICDI 1.2 is capable of causing significant disruption of CDI in the context of Cav1.3 (Fig. 4E-I) (27). This selective effect of ICDI 1.2 on Cav1.3 channels is intriguing as it represents a difficult to achieve discrimination between Cav1.2 and Cav1.3 (55,56). The strong homology between these two channels and has resulted in challenges to dissecting the contribution of each channel to the function of cells which express both channel subtypes, and hinders therapeutic options for neuropsychiatric disorders which may benefit from blockade of Cav1.3 (55). As such, the interface defined in this study between the IQ-A region and ICDI may represent a promising interface with which to selectively target Cav1.3.

The DCT of Cav1.2 is routinely cleaved in neurons and myocytes, with the cleavage product able to either remain associated with the channel (33), or translocate to the nucleus (41). Our FRET binding data would argue that this association between Cav1.2 and the DCT may be relatively weak, leaving $\mathrm{DCT}_{1.2}$ available to other binding partners. Moreover, it has been shown that an alternate start site exists within the C-tail of Cav1.2, such that alternative transcription of Cav1.2 will produce a DCT peptide containing a calcium channel associated transcription regulator (CCAT) (41). Importantly, ICDI would be intact within this peptide, providing an additional source of DCT $_{1.2}$ within cells. Our FRET binding analysis (Fig. 4A) suggests that the Cav1.2 DCT is capable of binding upstream calmodulatory elements in Cav1.2, albeit weakly. Functional analysis, however, suggests only minimal effects of this segment on Cav1.2 CDI. By comparison, the Cav1.343s channel variant contains all the elements required for high affinity binding with $\mathrm{DCT}_{1.2}$ and exhibits functional inhibition of CDI (Fig. 4G), consistent with previous studies (27). Thus, it seems likely that $\mathrm{DCT}_{1.2}$ may interact with this channel, altering the normally robust CDI. It is interesting to note, however, that while $\mathrm{DCT}_{1.2}$ is poised to modulate some Cav1.3 channels, the same cannot be said of $\mathrm{DCT}_{1.3}$. Not only is the IQ-A region of Cav1.2 suboptimal for binding 
to ICDI, but there is little evidence that Cav1.3 is cleaved in neurons (31). Thus, this cross-channel modulation may be unidirectional. Finally, since Cav1.2 and Cav1.3 often exist within the same neuron, this mode of cross-channel modulation may represent an important method for tuning CDI in different regions of the brain.

\section{Experimental procedures}

Molecular biology. The rat brain Cav1.3 a1 subunit (in pcDNA6) corresponds to AF370009.1 (57), and was incorporated to the mammalian expression plasmid pCDNA6 (Invitrogen) (20). This plasmid features a unique Bg/ll restriction site at a locus corresponding to $\sim 450$ amino acids upstream of the IQ domain, and a unique Xbal site after the stop codon which were used for generation of mutant and chimeric plasmids as descried below. The Cav1.2 a1 subunit (in pGW) is identical to rabbit NM001136522 (58), and the Cav1.4 channel (in pcDNA3) is the human clone corresponding to NP005174.2. Cav1.3 $3_{\triangle / \mathrm{DCT} 1.4}$ was made by fusing with the DCT of the Cav1.4 $\alpha 1$ subunit to the Cav1.3 a1 subunit (truncated after the IQ domain), as previously described (20). Cav1.343s was made by PCR amplification of the channel segment between the Bg/ll site and IQ domain with the appendage of amino acids unique to this splice variant (27). The PCR product was then inserted into the channel via the $\mathrm{Bg} / \mathrm{II} / \mathrm{Xbal}$ sites.

FRET constructs were fluorescent-tagged (either Venus or Cerulean) using similar strategies as previously described (45). Briefly, Venus and Cerulean fluorophores (a kind gift from Dr. Steven Vogel, NIH) were subcloned into the pcDNA3 vector via unique Kpnl and Notl sites. The PCRamplified channel peptides, as described in Liu et. al., were then cloned in via unique Notl and Xbal sites (20). Mutations were introduced into the channel or FRET construct via PCR amplification or overlap extension PCR.

Transfection of HEK293 cells. For electrophysiology experiments, HEK293 cells were cultured on 10-cm plates, and channels were transiently transfected by a calcium phosphate protocol (10). We applied $8 \mu \mathrm{g}$ of plasmid DNA encoding the desired pore forming a1 subunit, as well as $8 \mu \mathrm{g}$ of $\beta_{2 A}$ (M80545) and $8 \mu \mathrm{g}$ of rat $\alpha_{2 \delta}$ (NM012919.2) subunits along with $3 \mu \mathrm{g}$ of SV40 T antigen. For microscope-based FRET assays, HEK293 cells cultured on 3.5-cm culture dishes with integral No. 0 glass coverslip bottoms (In Vitro Scientific) were transiently transfected using polyethylenimine (PEI) reagent (Polysciences).

Whole-cell patch clamp recordings. Whole-cell recordings were obtained using an Axopatch 200A amplifier (Axon Instruments). Electrodes were pulled from borosilicate glass capillaries (Word Precision Instruments), with 1-3 M $\Omega$ resistances, which were in turn compensated for series resistance by $>60 \%$. Currents were low-pass filtered at $2 \mathrm{kHz}$ before digital acquisition at five times the frequency. A P/8 leak subtraction protocol was used. The internal solution contained (in mM): CsMeSO3, 114; CsCl, 5; MgATP, 4; HEPES (pH 7.4), 10; and BAPTA (1,2-bis(oaminophenoxy)ethane- $N, N, N^{\prime}, N^{\prime}$-tetraacetic acid), 10; at 295 mOsm adjusted with CsMeSO3. The bath solution contained (in mM): TEA-MeSO3, 102; HEPES ( $\mathrm{pH} \mathrm{7.4),} \mathrm{10;} \mathrm{CaCl} 2$ or $\mathrm{BaCl}$, 40; at 305 mOsm adjusted with TEA-MeSO3. Data was analyzed using custom Matlab scripts. Inactivation was quantified as the ratio of current remaining after $300 \mathrm{~ms}$ in either $\mathrm{Ca}^{2+}$ or $\mathrm{Ba}^{2+}$ $\left(r_{300}\right)$, allowing CDI to be quantified as the difference between the $r_{300}$ in $\mathrm{Ba}^{2+}$ vs $\mathrm{Ca}^{2+}$, measured at $10 \mathrm{mV}$ for Cav1.3 channels, and $30 \mathrm{mV}$ cof Cav1.2. 
FRET optical imaging. FRET two-hybrid experiments were performed on an inverted microscope as described $(45,46)$. The bath solution was a Tyrode's solution composed of (in $\mathrm{mM}$ ): $\mathrm{NaCl}, 138$; $\mathrm{KCl}, 4 ; \mathrm{MgCl} 2$, 1; HEPES (pH 7.4), 10; $\mathrm{CaCl} 2$, 2; at 305 mOsm adjusted with glucose. Background fluorescent signals were measured from cells without expression of the fluorophores and subtracted from cells expressing the fluorophores. Concentration-dependent spurious FRET was subtracted from the raw data prior to binding-curve analysis $(45,46)$. Cerulean (59) and Venus (60) were used as the donor and acceptor fluorescent proteins instead of eCFP and eYFP, as their optical properties provided more robust and stable FRET signals. Acceptor-centric measurements of FRET were obtained with the $3^{3}$-FRET algorithm $(45,46)$, in which the effective FRET efficiency $\left(E_{\mathrm{EFF}}\right)$ and FRET ratio $(F R)$ are defined as:

$$
E_{\mathrm{Eff}}=E \times A_{\mathrm{b}}=(F R-1) \cdot\left[\varepsilon_{\mathrm{Ven}(440 \mathrm{~nm})} / \varepsilon_{\mathrm{Cer}(440 \mathrm{~nm})}\right]
$$

where $E$ is the FRET efficiency of a donor-acceptor pair, $A_{\mathrm{b}}$ is the fraction of acceptor molecules bound by a donor, and $\varepsilon_{V e n}(440 \mathrm{~nm}) / \varepsilon_{\mathrm{Cer}(440 \mathrm{~nm})}$ is the approximate molar extinction coefficients of Cerulean and Venus, which was measured as 0.08 on our setup. Intensity measurements at each wavelength were measured from individual cells such that variable expression across the cells enabled population of a binding curve. Binding curves were analyzed using GraphPad software (Prism), providing relative $K_{d}$ values and standard error based on an unbiased the fit to the data. These relative $K_{d}$ values were then calibrated according to a previously determined calibration factor $(13,20)$, and converted to $K_{\mathrm{a}}=1 / K_{\mathrm{d}}$. Importantly, the previous calibration factor determined for our setup utilized CFP/YFP FRET pairs. In order to account for the difference in FR using cerulean and venus, we determined the relative $K_{d}$ for multiple peptides using both the florouphore pairs and found that the two data sets differed by a factor of 1.8 , which we incorporated into the calibration factor.

\section{Data availability}

All data is contained within the manuscript.

\section{Acknowledgements}

This project was initiated under the direction of Dr. David Yue, who passed away in 2014. David was a brilliant scientist and exceptional mentor, and we are grateful for his guidance and friendship. We would like to thank Dr. Gordon Tomaselli for his guidance and support. We would also like to thank Hojjat Bazzazi for providing FRET plasmids, and for useful discussion and advice throughout the project. In addition, we thank Wanjun Yang for dedicated technical support and thank members of the Calcium Signals lab at Johns Hopkins for discussion and support of this project, as well as members of Dr. Dick's lab at the University of Maryland for insightful discussions and editing of the manuscript.

\section{Funding and additional information}

This grant was supported by an NIH/NINDS grant 5R01NS085074 and by an NIH/NHLBI grant 1R01HL149926.

\section{Conflict of interest}

The authors declare that they have no conflicts of interest with the contents of this article. 


\section{Abbreviations and nomenclature}

$\begin{array}{ll}\text { CaM } & \text { calmodulin } \\ \text { apoCaM } & \text { Ca }^{2+} \text { free calmodulin } \\ \text { calcium/CaM dependent inactivation } \\ \text { Cav } & \text { voltage gated calcium channel } \\ \text { Ca }^{2+} & \text { calcium } \\ \text { FRET } & \text { fluorescence resonance energy transfer } \\ \text { FR } & \text { fret ratio } \\ \text { iTL } & \text { individually transformed Langmuir } \\ \text { CFP } & \text { cerulean fluorescent protein } \\ \text { YFP } & \text { yellow fluorescent protein } \\ \text { DCT } & \text { distal carboxy tail } \\ \text { ICDI } & \text { inhibitor of CDI } \\ \text { PCRD } & \text { proximal C-terminal regulatory domain } \\ \text { DCRD } & \text { distal C-terminal regulatory domain }\end{array}$




\section{Figure legends}

Figure 1. Identifying the hotspots on the IQ region required for ICDI binding and function (A) Cartoon depicting the chimeric Cav1.3 $3_{\triangle \mathrm{DCT} 1.4}$ channel. (B) Exemplar whole cell recording showing minimal inactivation in response to a $10 \mathrm{mV}$ step depolarization in $\mathrm{Ba}^{2+}$ (black) or $\mathrm{Ca}^{2+}$ (red) for Cav1.3 ${ }_{\triangle \mathrm{DCT} 1.4}$ demonstrating the function of $\mathrm{ICDI}_{1.4}$. $\mathrm{Ba}^{2+}$ current is scaled to enable comparison of the kinetics of the two traces. Scale bar refers to the $\mathrm{Ca}^{2+}$ trace here and throughout. (C) Exemplar whole cell recording of the truncated Cav1.3 channel, lacking the DCT containing ICDI. Robust CDI is seen as the strong decay of the $\mathrm{Ca}^{2+}$ current (red) as compared to the $\mathrm{Ba}^{2+}$ current. (D) FRET two-hybrid assay of IQ/ICDI interaction. FRET binding partners are displayed on the left, with star indicating the locus of alanine mutations. Strong binding was measured for wild-type prelQ $\mathrm{Q}_{3}-\mathrm{IQ}_{-} \mathrm{A}_{1.3}$ with $\mathrm{ICDI}_{1.4}$ (black), while the mutation $\mathrm{F}[-2] \mathrm{A}$ (blue) decreased the binding affinity compared to WT (gray). (E) Summary of $K_{a}$ values for mutant Venus-preIQ $-I Q-A_{1.3}$ versus Cerulean-ICDI ${ }_{1.4}$ peptides measured with FRET two-hybrid assay as in panel $D$. Alanine was systematically substituted into the IQ region of the Venus-prelQ $Q_{3}-I Q-A_{1.3}$ peptide, with the identity of the amino acid displayed on the top and bottom of the bar graph such that the canonical ' $I$ ' of the IQ region is given position 0 . The dashed line indicates the WT value and the blue bar corresponds to F[-2]A displayed in panel D. Data are displayed as mean \pm SEM. (F) Exemplar patch clamp data corresponding to F[-2]A in Cav1.3 $3_{\triangle / \text { DCT1.4 }}$ demonstrating a partial recovery of $\mathrm{CDI}$ due to the mutation. (G) Average $\mathrm{CDI}$ values for each mutation, colors correspond to the data in other panels. $(\mathbf{H}) \mathrm{CDI}$ and binding data for the mutations is well fit by equation 2 , validating the competitive mechanism. Colored circles correspond to the colored data in the figure panels.

\section{Figure 2. Identifying the hotspots on ICDI $_{1.4}$ required for ICDI binding and function}

(A) FRET binding partners are displayed on the left, with the star indicating the locus of alanine

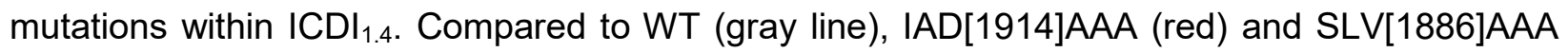
(green) significantly decreased binding affinity between the FRET pairs to differing degrees. (B) Summary of $K_{a}$ values for Venus-PrelQ ${ }_{3}-I Q A_{1.3}$ versus mutant Cerulean-ICDI ${ }_{1.4}$ peptides measured with FRET two-hybrid as in panel A. Alanine was systematically substituted three amino acids at a time into Cerulean-ICDI 1.4 , with the identity of the amino acids displayed on the top of the bar graph. Amino acids labeled in gray (KQE, YSD) were not evaluated due to poor expression. The dashed line indicates the WT value and the colored bars correspond to data in other panels of the figure here and throughout. Data are displayed \pm SEM. (C) Summary of CDI values measured for Cav1.3 ${ }_{\triangle / \mathrm{DCT} 1.4}$ harboring mutations corresponding to mutations which produced large effects on binding affinity in $\mathbf{B}$. Gray dashed line represents the robust CDI of the channel without $\mathrm{ICDI}_{1.4}$, while the black dashed line indicates the nominal CDI of WT

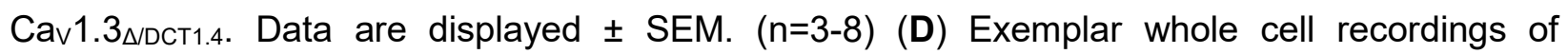
Cav1.3 $\triangle$ IDCT1.4, demonstrating a significant restoration of CDI due to IAD[1914]AAA and near complete CDI due to SLV[1886]AAA. (E) Robust fit of CDI (C) and FRET binding data (B) to equation 2, using identical parameters as in Figure $1 \mathrm{H}$. Light gray circles indicate data from Figure 1, while dark gray and colored circles indicate mutations within ICDI corresponding to $\mathbf{B}$ and $\mathbf{C}$, with green and red circles indicating exemplars shown in other panels for reference. The PKA phosphorylation site Ser1883 is plotted as the orange circle. 
Figure 3. Elucidating the role of the A region for ICDI interaction.

(A) Cartoon depicting FRET interacting pairs for panels $\mathbf{B}$ and $\mathbf{C}$. Portions of the PrelQ $\mathbf{Q}_{3}-\mathrm{IQ}_{\mathbf{Q}} \mathrm{A}_{1.3}$ were paired with $I C D I_{1.4}$ to identify critical regions. (B) IQ-A $A_{1.3}$ is sufficient to support robust FRET (blue), indicating that the pre- $I Q_{3}$ region is not involved in the interaction. However, the $A$ region alone is not sufficient to support binding (open circles). (C) $K_{\mathrm{a}}$ values for each channel fragment indicate that neither $I Q_{1.3}$ or $A_{1.3}$ is sufficient for robust binding with $I C D I_{1.4}$, however both regions are necessary as indicated by the robust binding of IQ- $\mathrm{A}_{1.3}$ with $I C D I_{1.4}$. Data are displayed as mean \pm SEM here and throughout. (D) Cartoon depicting FRET interacting pairs for panels $\mathbf{E}$ and F. Cerulean-ICDI ${ }_{1.4}$ was paired with various truncations of Venus-PrelQ $Q_{3}-I Q_{2}-A_{1.3}$. the blue box shows the sequence of the $A$ region and illustrates the truncation strategy. (E, F) Truncating up to 34 amino acids distal to the IQ region $(\Delta 34)$ had nominal effects on binding (green), however removal of 6 additional amino acids $(\Delta 28)$ dramatically reduced binding affinity (red). (G) Cartoon depicting FRET interacting pairs for panels $\mathbf{H}$ and $\mathbf{I}$. Alanine substitutions were made within the A region, as indicated by the yellow star. (H) Mutation of KPY[1626]AAA within the A region had no effect on binding (cyan), while ALQ[1635]AAA (purple) diminished the binding affinity between $I Q-A_{1.3}$ and $I C D I_{1.4}$. (I) Summary of $K_{a}$ values for alanine mutations in the A region indicate multiple critical amino acids.

\section{Figure 4. Residual functionality of ICDI1.2 in modulating Cav channels.}

(A) Cartoon depicting FRET interacting pairs for panels $B$ and C. Venus-PrelQ $Q_{3}-I_{Q}-A_{1.2}$ or VenusPrelQ $Q_{3}-I Q_{2} A_{1.3}$ was paired with Cerulean-ICDI 1.2 in order to evaluate the role of the ICDI contained within Cav1.2 channels. (B) Venus-PrelQ ${ }_{3}-I_{Q}-A_{1.2}$ displays moderate binding with Cerulean$\mathrm{ICDI}_{1.2}$ (blue) as compared to the robust binding between Venus-PrelQ $\mathrm{Q}_{3}-\mathrm{IQ}_{-}-\mathrm{A}_{1.3}$ and Cerulean$I C D I_{1.4}$ which is reproduced as the gray line for comparison. (C) Venus-PrelQ ${ }_{3}-I_{Q}-A_{1.3}$ is able to bind strongly with Cerulean-ICDI ${ }_{1.2}$. (D) Left: channel cartoon with cleavage site indicated by the yellow arrowhead. Right: Exemplar data demonstrating that truncation of Cav1.2 at the cleavage site has a minor effect on CDI. (E) Left: channel cartoon indicating introduction of ICDI1.2 into the Cav1.3 channel backbone. Right: Introduction of $\mathrm{ICDI}_{1.2}$ into Cav1.3 channels causes a large decrease in CDI. (F) Average CDI data demonstrating a modest but statistically significant effect of $\mathrm{ICDI}_{1.2}$ on Cav1.2 channels (blue), and a large effect on Cav1.3 which is comparable to the native effect of ICDI1.3 contained within Cav1.3 long (gray). Data are plotted as mean \pm SEM. $\left({ }^{*} p \leq 0.05,{ }^{* *} p \leq 0.01\right)(\mathbf{G})$ Left: cartoon of human Cav1.343s, with the sequence of the end of the channel displayed below. This splice variant contains a portion of the A region (blue highlight) which contains the identified hotspots required for ICDI binding (bold), as well as a short sequence of unique amino acids prior to truncation of the channel (pink). Right: exemplar whole cell patch clamp data demonstrates robust CDI in WT Cav1.343s, which is reduced when the predicted cleavage fragment of the human Cav1.2 channel is co-expressed, or when ICDI 1.4 is expressed as a peptide. $(\mathbf{H})$ Average $\mathrm{CDI}$ effects for the channels described in panel $\mathrm{G}, \mathrm{DCT}_{1.2}$ and $\mathrm{ICDI}_{1.4}$ both have significant effects on the CDI of Cav1.343s ( ${ }^{* *} p \leq 0.01$, data are displayed \pm SEM). (I) Cartoon proposing a cross-channel effect of $\mathrm{ICDI}_{1.2}$, such that cleaved or independently transcribed $\mathrm{DCT}_{1.2}$ may modulate Cav1.343s channels. 

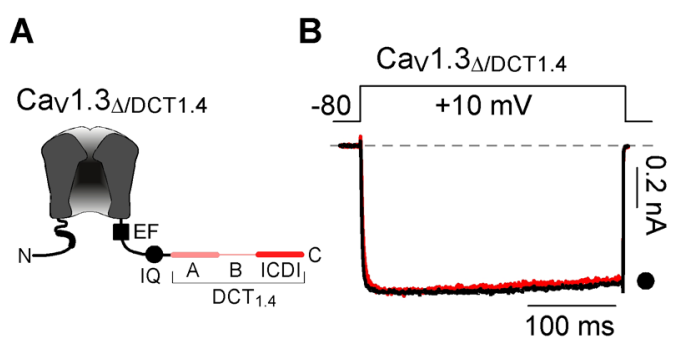

D

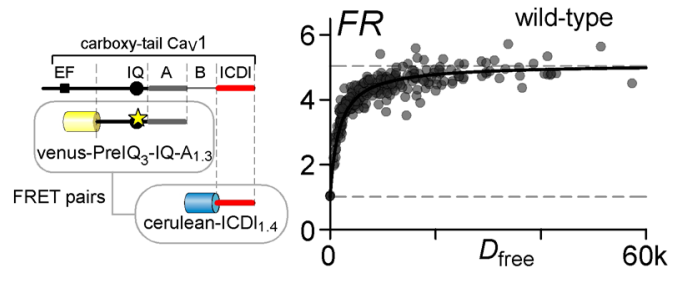

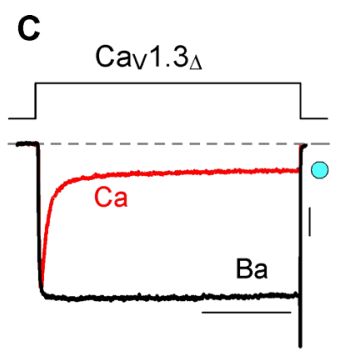

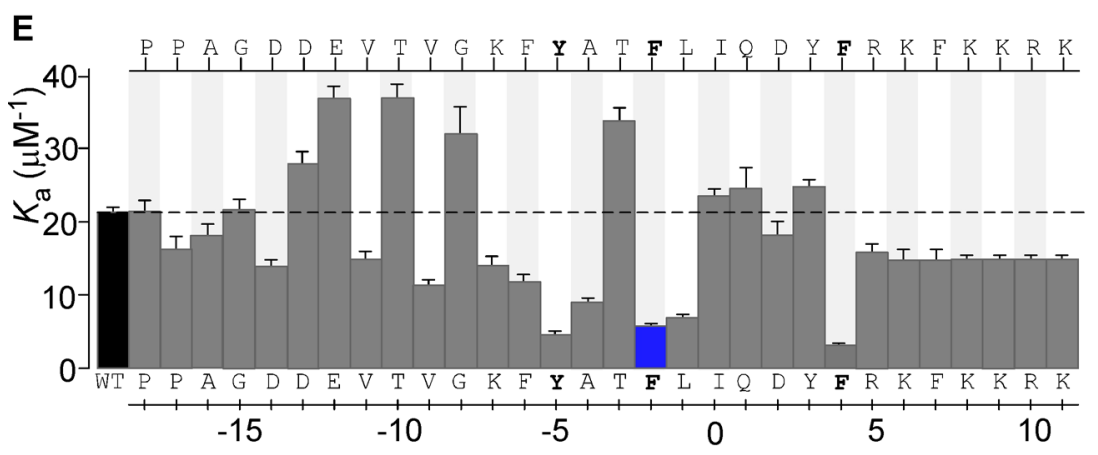

F
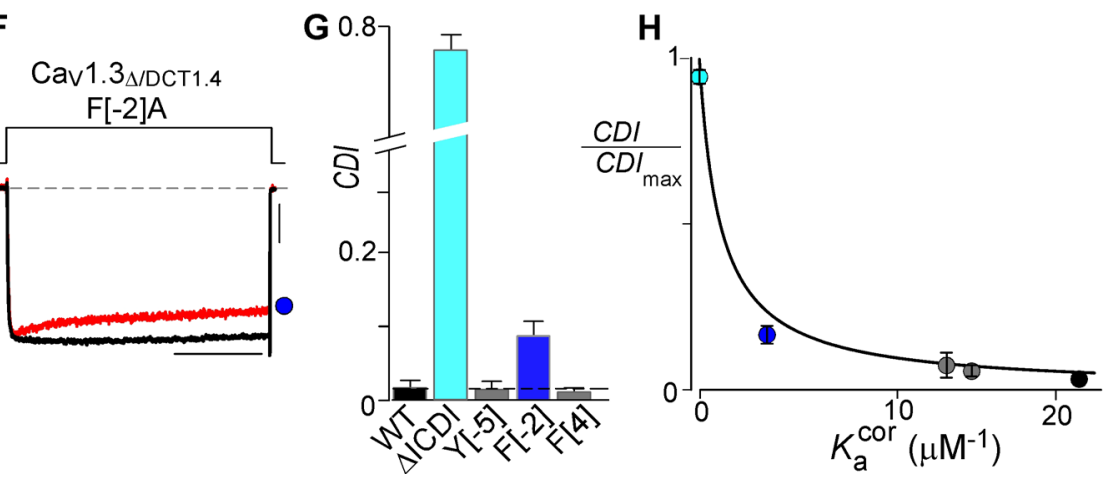

Figure 1 
A

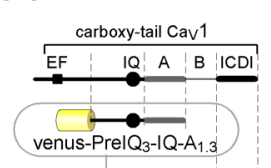

FRET pairs
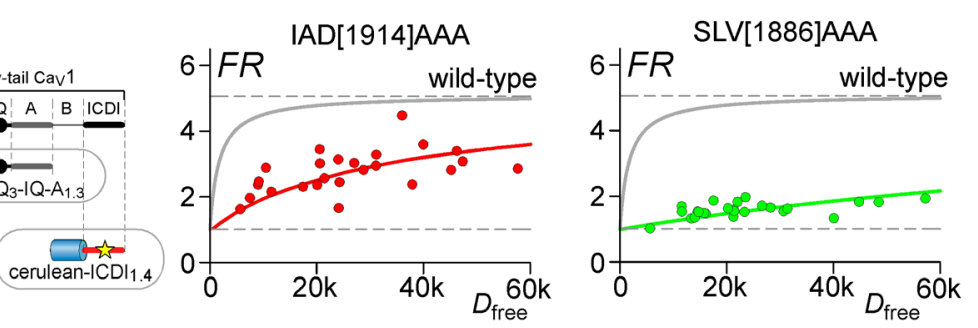

B

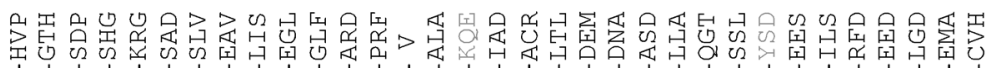

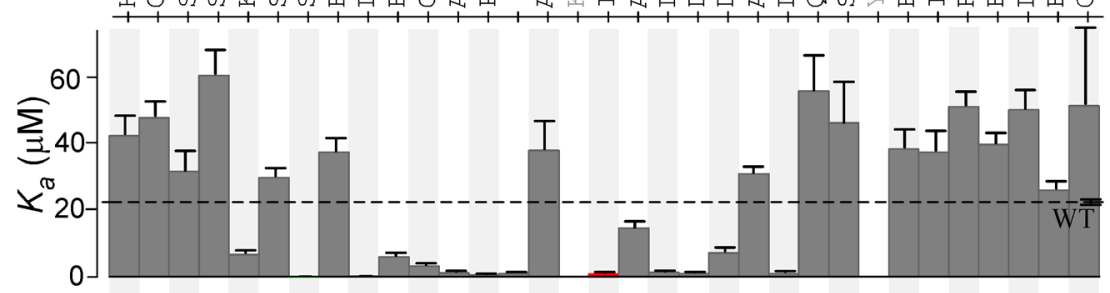

C

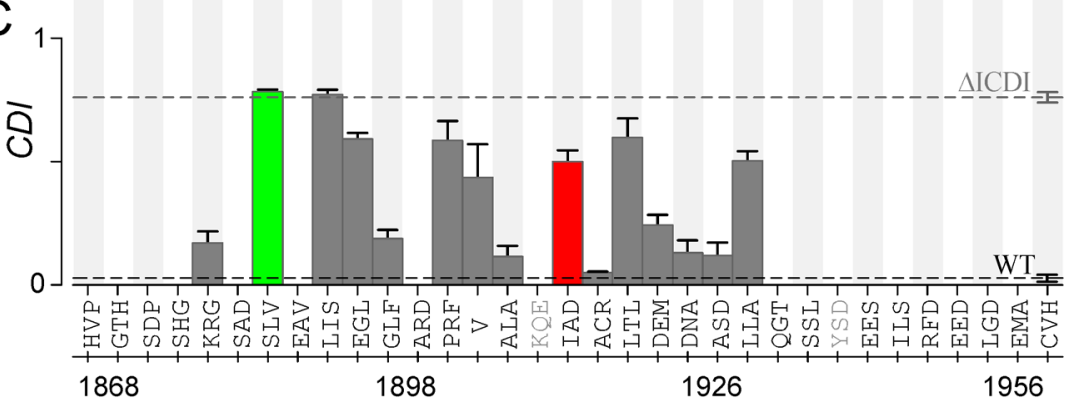

D

E
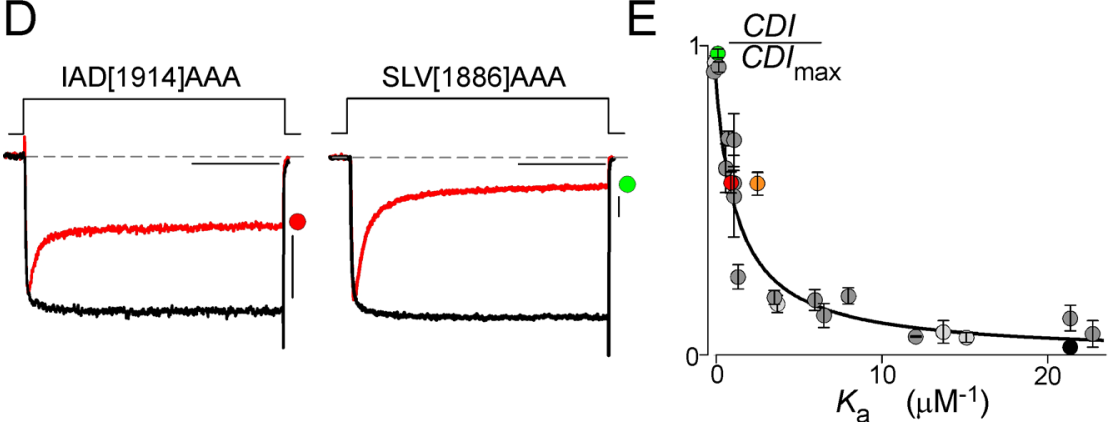

Figure 2 
A
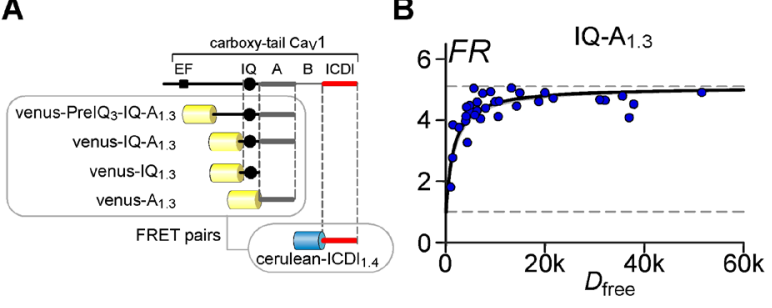

D

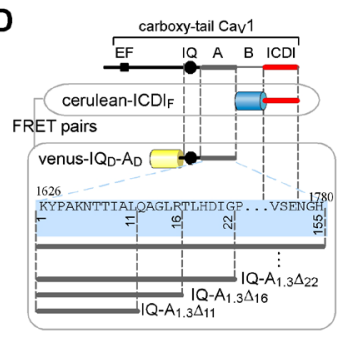

G

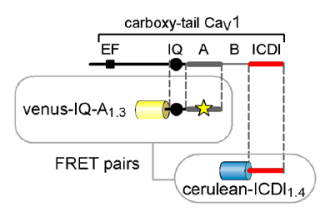

E

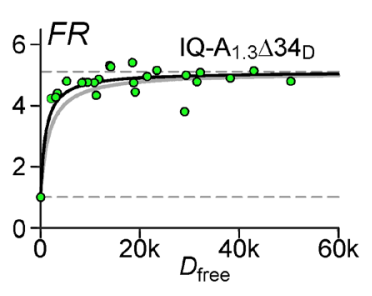

H

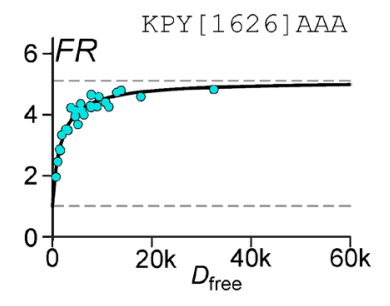

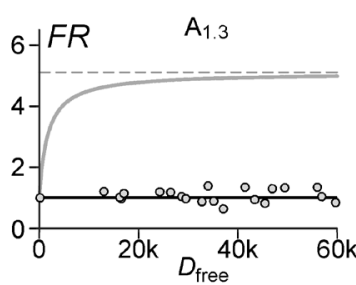
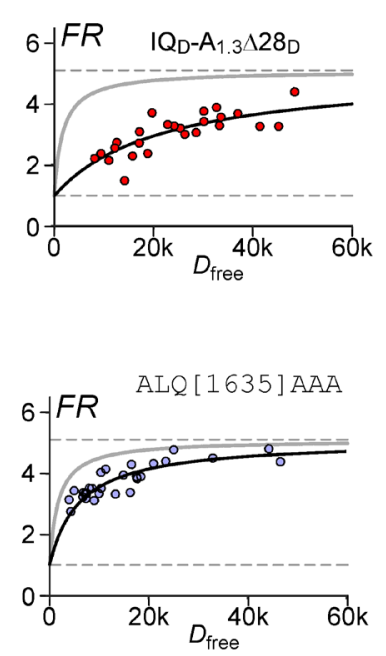

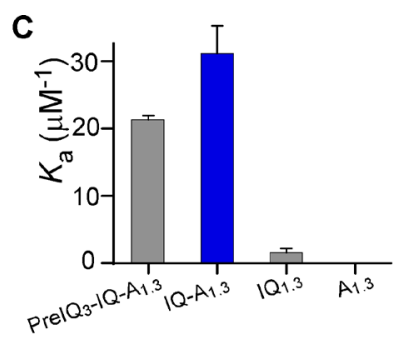

F
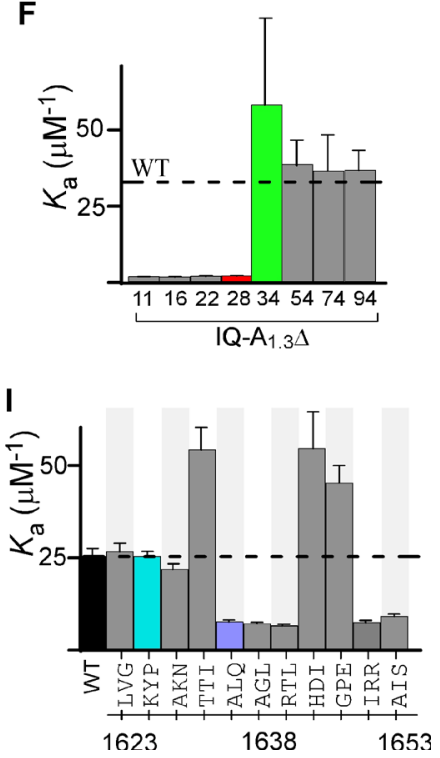

Figure 3 

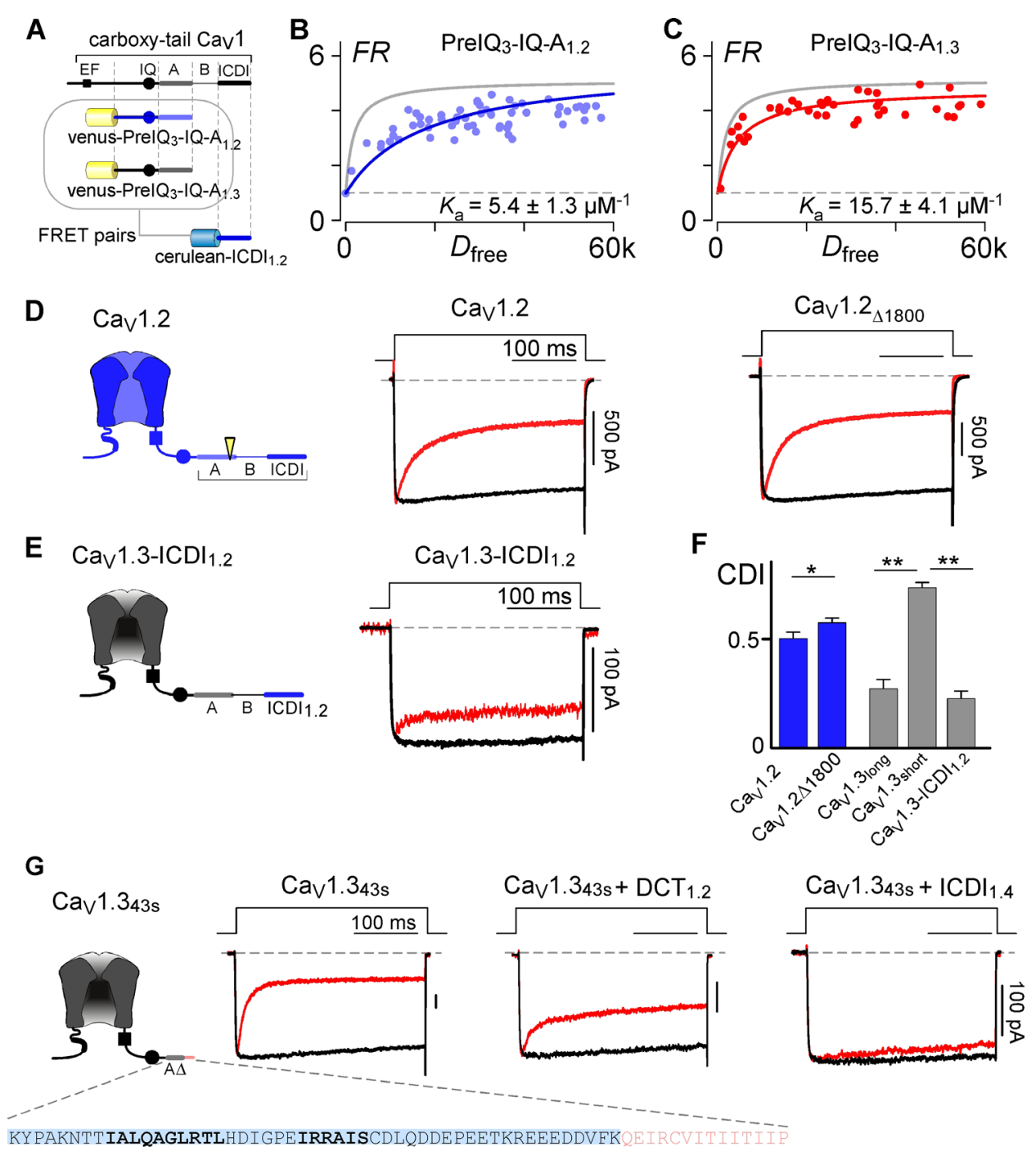

H

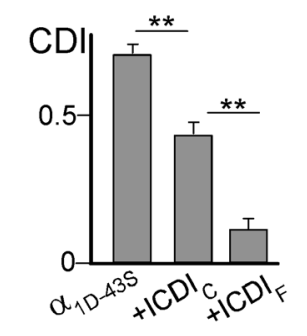

I

Cav1.343s

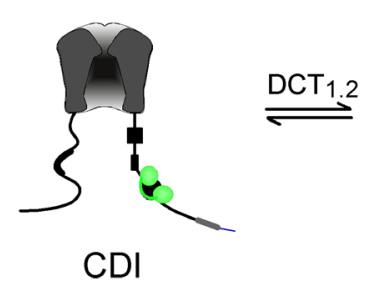

Cav1.343s

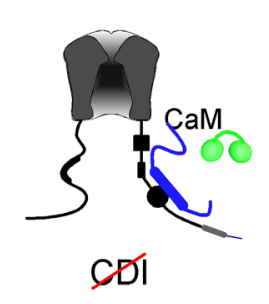

Figure 4 


\section{References}

1. Harvey, R. D., and Hell, J. W. (2013) CaV1.2 signaling complexes in the heart. J Mol Cell Cardiol 58, 143-152

2. Gutierrez, L. M., Brawley, R. M., and Hosey, M. M. (1991) Dihydropyridine-sensitive calcium channels from skeletal muscle. I. Roles of subunits in channel activity. J Biol Chem 266, 16387-16394

3. Striessnig, J., Koschak, A., Sinnegger-Brauns, M. J., Hetzenauer, A., Nguyen, N. K., Busquet, P., Pelster, G., and Singewald, N. (2006) Role of voltage-gated L-type Ca2+ channel isoforms for brain function. Biochem Soc Trans 34, 903-909

4. Lipscombe, D., Helton, T. D., and Xu, W. (2004) L-type calcium channels: the low down. J Neurophysiol 92, 2633-2641

5. McDonald, T. F., Pelzer, S., Trautwein, W., and Pelzer, D. J. (1994) Regulation and modulation of calcium channels in cardiac, skeletal, and smooth muscle cells. Physiol Rev 74, 365-507

6. Ghosh, D., Syed, A. U., Prada, M. P., Nystoriak, M. A., Santana, L. F., Nieves-Cintron, M., and Navedo, M. F. (2017) Calcium Channels in Vascular Smooth Muscle. Adv Pharmacol 78, 49-87

7. Liang, H., DeMaria, C. D., Erickson, M. G., Mori, M. X., Alseikhan, B. A., and Yue, D. T. (2003) Unified mechanisms of $\mathrm{Ca}^{2+}$ regulation across the $\mathrm{Ca}^{2+}$ channel family. Neuron 39, 951-960

8. Pitt, G. S., Zuhlke, R. D., Hudmon, A., Schulman, H., Reuter, H., and Tsien, R. W. (2001) Molecular basis of calmodulin tethering and Ca2+-dependent inactivation of Ltype Ca2+ channels. J. Biol. Chem. 276, 30794-30802

9. Alseikhan, B. A., DeMaria, C. D., Colecraft, H. M., and Yue, D. T. (2002) Engineered calmodulins reveal the unexpected eminence of $\mathrm{Ca} 2+$ channel inactivation in controlling heart excitation. Proceedings of the National Academy of Sciences of the United States of America 99, 17185-17190

10. Peterson, B. Z., DeMaria, C. D., Adelman, J. P., and Yue, D. T. (1999) Calmodulin is the $\mathrm{Ca}^{2+}$ sensor for Ca2+ -dependent inactivation of L- type calcium channels. Neuron 22, 549-558

11. Zuhlke, R. D., Pitt, G. S., Deisseroth, K., Tsien, R. W., and Reuter, H. (1999) Calmodulin supports both inactivation and facilitation of L-type calcium channels. Nature 399, 159162

12. Adams, P. J., Ben-Johny, M., Dick, I. E., Inoue, T., and Yue, D. T. (2014) Apocalmodulin itself promotes ion channel opening and $\mathrm{Ca}(2+)$ regulation. Cell 159, 608-622

13. Bazzazi, H., Ben Johny, M., Adams, P. J., Soong, T. W., and Yue, D. T. (2013) Continuously tunable $\mathrm{Ca}(2+)$ regulation of RNA-edited $\mathrm{CaV} 1.3$ channels. Cell reports $\mathbf{5}$, 367-377

14. Ben Johny, M., Yang, P. S., Bazzazi, H., and Yue, D. T. (2013) Dynamic switching of calmodulin interactions underlies $\mathrm{Ca} 2+$ regulation of $\mathrm{CaV} 1.3$ channels. Nature communications 4, 1717

15. Splawski, I., Timothy, K. W., Decher, N., Kumar, P., Sachse, F. B., Beggs, A. H., Sanguinetti, M. C., and Keating, M. T. (2005) Severe arrhythmia disorder caused by cardiac L-type calcium channel mutations. Proceedings of the National Academy of Sciences of the United States of America 102, 8089-8096; discussion 8086-8088

16. Splawski, I., Timothy, K. W., Sharpe, L. M., Decher, N., Kumar, P., Bloise, R., Napolitano, C., Schwartz, P. J., Joseph, R. M., Condouris, K., Tager-Flusberg, H., Priori, S. G., Sanguinetti, M. C., and Keating, M. T. (2004) Ca(V)1.2 calcium channel dysfunction causes a multisystem disorder including arrhythmia and autism. Cell 119, 19-31 
17. Griessmeier, K., Cuny, H., Roetzer, K., Griesbeck, O., Harz, H., Biel, M., and WahlSchott, C. (2009) Calmodulin is a functional regulator of Cav1.4 I-type Ca ${ }^{2+}$ channels. $J$ Biol Chem

18. Haeseleer, F., Williams, B., and Lee, A. (2016) Characterization of C-terminal Splice Variants of Cav1.4 Ca2+ Channels in Human Retina. J Biol Chem 291, 15663-15673

19. Yang, P. S., Alseikhan, B. A., Hiel, H., Grant, L., Mori, M. X., Yang, W., Fuchs, P. A., and Yue, D. T. (2006) Switching of $\mathrm{Ca}^{2+}$-dependent inactivation of Cav1.3 channels by calcium binding proteins of auditory hair cells. J. Neurosci. 26, 10677-10689

20. Liu, X., Yang, P. S., Yang, W., and Yue, D. T. (2010) Enzyme-inhibitor-like tuning of $\mathrm{Ca}(2+)$ channel connectivity with calmodulin. Nature 463, 968-972

21. Yang, P. S., and Yue, D. T. (2009) Insights into mutations in calcium binding protein 4, CaBP4, that cause incomplete type congenital stationary night (abstr.) Society for Neuroscience 2009

22. Shaltiel, L., Paparizos, C., Fenske, S., Hassan, S., Gruner, C., Rotzer, K., Biel, M., and Wahl-Schott, C. A. (2012) Complex regulation of voltage-dependent activation and inactivation properties of retinal voltage-gated Cav1.4 L-type Ca2+ channels by $\mathrm{Ca} 2+-$ binding protein 4 (CaBP4). J Biol Chem 287, 36312-36321

23. Campiglio, M., Coste de Bagneaux, P., Ortner, N. J., Tuluc, P., Van Petegem, F., and Flucher, B. E. (2018) STAC proteins associate to the IQ domain of CaV1.2 and inhibit calcium-dependent inactivation. Proceedings of the National Academy of Sciences of the United States of America 115, 1376-1381

24. Niu, J., Dick, I. E., Yang, W., Bamgboye, M. A., Yue, D. T., Tomaselli, G., Inoue, T., and Ben-Johny, M. (2018) Allosteric regulators selectively prevent $\mathrm{Ca}(2+)$-feedback of $\mathrm{CaV}$ and $\mathrm{NaV}$ channels. Elife 7

25. Polster, A., Dittmer, P. J., Perni, S., Bichraoui, H., Sather, W. A., and Beam, K. G. (2018) Stac Proteins Suppress $\mathrm{Ca}(2+)$-Dependent Inactivation of Neuronal I-type $\mathrm{Ca}(2+)$ Channels. The Journal of neuroscience : the official journal of the Society for Neuroscience 38, 9215-9227

26. Wong King Yuen, S. M., Campiglio, M., Tung, C. C., Flucher, B. E., and Van Petegem, F. (2017) Structural insights into binding of STAC proteins to voltage-gated calcium channels. Proceedings of the National Academy of Sciences of the United States of America 114, E9520-E9528

27. Bock, G., Gebhart, M., Scharinger, A., Jangsangthong, W., Busquet, P., Poggiani, C., Sartori, S., Mangoni, M. E., Sinnegger-Brauns, M. J., Herzig, S., Striessnig, J., and Koschak, A. (2011) Functional Properties of a Newly Identified C-terminal Splice Variant of Cav1.3 L-type Ca2+ Channels. J Biol Chem 286, 42736-42748

28. Tan, G. M., Yu, D., Wang, J., and Soong, T. W. Alternative Splicing at C-terminus of CaV1.4 Calcium Channel Modulates Calcium-Dependent Inactivation, Activation Potential and Current Density. J Biol Chem

29. Wahl-Schott, C., Baumann, L., Cuny, H., Eckert, C., Griessmeier, K., and Biel, M. (2006) Switching off calcium-dependent inactivation in L-type calcium channels by an autoinhibitory domain. Proceedings of the National Academy of Sciences of the United States of America 103, 15657-15662

30. Lieb, A., Scharinger, A., Sartori, S., Sinnegger-Brauns, M. J., and Striessnig, J. (2012) Structural determinants of CaV1.3 L-type calcium channel gating. Channels (Austin) 6, 197-205

31. Singh, A., Gebhart, M., Fritsch, R., Sinnegger-Brauns, M. J., Poggiani, C., Hoda, J. C., Engel, J., Romanin, C., Striessnig, J., and Koschak, A. (2008) Modulation of voltageand $\mathrm{Ca}^{2+}$-dependent gating of Cav1.3 L-type calcium channels by alternative splicing of a C-terminal regulatory domain. J. Biol. Chem. 283, 20733-20744 
32. Singh, A., Hamedinger, D., Hoda, J. C., Gebhart, M., Koschak, A., Romanin, C., and Striessnig, J. (2006) C-terminal modulator controls $\mathrm{Ca}^{2+}$-dependent gating of Cav1.4 Ltype $\mathrm{Ca}^{2+}$ channels. Nat Neurosci 9, 1108-1116

33. Hulme, J. T., Yarov-Yarovoy, V., Lin, T. W., Scheuer, T., and Catterall, W. A. (2006) Autoinhibitory control of the CaV1.2 channel by its proteolytically processed distal Cterminal domain. The Journal of physiology 576, 87-102

34. Christel, C. J., Cardona, N., Mesirca, P., Herrmann, S., Hofmann, F., Striessnig, J., Ludwig, A., Mangoni, M. E., and Lee, A. (2012) Distinct localization and modulation of Cav1.2 and Cav1.3 L-type Ca2+ channels in mouse sinoatrial node. The Journal of physiology 590, 6327-6342

35. Mangoni, M. E., Couette, B., Bourinet, E., Platzer, J., Reimer, D., Striessnig, J., and Nargeot, J. (2003) Functional role of L-type Cav1.3 Ca2+ channels in cardiac pacemaker activity. Proceedings of the National Academy of Sciences of the United States of America 100, 5543-5548

36. Huang, H., Tan, B. Z., Shen, Y., Tao, J., Jiang, F., Sung, Y. Y., Ng, C. K., Raida, M., Kohr, G., Higuchi, M., Fatemi-Shariatpanahi, H., Harden, B., Yue, D. T., and Soong, T. W. (2012) RNA editing of the IQ domain in Cav1.3 channels modulates their $\mathrm{Ca}^{2+}-$ dependent inactivation. Neuron in press

37. Bezprozvanny, I. (2009) Calcium signaling and neurodegenerative diseases. Trends Mol Med 15, 89-100

38. Hurley, M. J., Brandon, B., Gentleman, S. M., and Dexter, D. T. (2013) Parkinson's disease is associated with altered expression of CaV1 channels and calcium-binding proteins. Brain 136, 2077-2097

39. Hoda, J. C., Zaghetto, F., Koschak, A., and Striessnig, J. (2005) Congenital stationary night blindness type 2 mutations S229P, G369D, L1068P, and W1440X alter channel gating or functional expression of $\mathrm{Ca}(\mathrm{v}) 1.4 \mathrm{~L}$-type Ca2+ channels. The Journal of neuroscience : the official journal of the Society for Neuroscience 25, 252-259

40. Strom, T. M., Nyakatura, G., Apfelstedt-Sylla, E., Hellebrand, H., Lorenz, B., Weber, B. H., Wutz, K., Gutwillinger, N., Ruther, K., Drescher, B., Sauer, C., Zrenner, E., Meitinger, T., Rosenthal, A., and Meindl, A. (1998) An L-type calcium-channel gene mutated in incomplete X-linked congenital stationary night blindness. Nat Genet 19, 260-263

41. Gomez-Ospina, N., Tsuruta, F., Barreto-Chang, O., Hu, L., and Dolmetsch, R. (2006) The $\mathrm{C}$ terminus of the L-type voltage-gated calcium channel $\mathrm{Ca}(\mathrm{V}) 1.2$ encodes a transcription factor. Cell 127, 591-606

42. Schroder, E., Byse, M., and Satin, J. (2009) L-Type Calcium Channel C Terminus Autoregulates Transcription. Circ Res 104, 1373-U1238

43. Sang, L., Dick, I. E., and Yue, D. T. (2016) Protein kinase A modulation of CaV1.4 calcium channels. Nature communications 7, 12239

44. Doering, C. J., Hamid, J., Simms, B., McRory, J. E., and Zamponi, G. W. (2005) Cav1.4 encodes a calcium channel with low open probability and unitary conductance. Biophys J 89, 3042-3048

45. Erickson, M. G., Alseikhan, B. A., Peterson, B. Z., and Yue, D. T. (2001) Preassociation of calmodulin with voltage-gated $\mathrm{Ca}(2+)$ channels revealed by FRET in single living cells. Neuron 31, 973-985

46. Erickson, M. G., Liang, H., Mori, M. X., and Yue, D. T. (2003) FRET two-hybrid mapping reveals function and location of L-type $\mathrm{Ca} 2+$ channel CaM preassociation. Neuron 39, 97-107

47. Liu, N., Yang, Y., Ge, L., Liu, M., Colecraft, H. M., and Liu, X. (2017) Cooperative and acute inhibition by multiple C-terminal motifs of L-type $\mathrm{Ca}(2+)$ channels. Elife 6 
48. Fuller, M. D., Emrick, M. A., Sadilek, M., Scheuer, T., and Catterall, W. A. (2010) Molecular Mechanism of Calcium Channel Regulation in the Fight-or-Flight Response. Sci Signal 3, ra70-ra70

49. Hulme, J. T., Konoki, K., Lin, T. W., Gritsenko, M. A., Camp, D. G., Bigelow, D. J., and Catterall, W. A. (2005) Sites of proteolytic processing and noncovalent association of the distal C-terminal domain of CaV1.1 channels in skeletal muscle. Proceedings of the National Academy of Sciences of the United States of America 102, 5274-5279

50. Chaudhuri, D., Issa, J. B., and Yue, D. T. (2007) Elementary Mechanisms Producing Facilitation of Cav2.1 (P/Q-type) Channels. J Gen Physiol 129, 385-401

51. Kim, J., Ghosh, S., Nunziato, D. A., and Pitt, G. S. (2004) Identification of the components controlling inactivation of voltage-gated $\mathrm{Ca}^{2+}$ channels. Neuron $41,745-754$

52. Lee, A., Zhou, H., Scheuer, T., and Catterall, W. A. (2003) Molecular determinants of $\mathrm{Ca}(2+) /$ calmodulin-dependent regulation of $\mathrm{Ca}(\mathrm{v}) 2.1$ channels. Proceedings of the National Academy of Sciences of the United States of America 100, 16059-16064

53. Gerhardstein, B. L., Gao, T., Bunemann, M., Puri, T. S., Adair, A., Ma, H., and Hosey, M. M. (2000) Proteolytic processing of the $C$ terminus of the alpha(1C) subunit of L-type calcium channels and the role of a proline-rich domain in membrane tethering of proteolytic fragments. J Biol Chem 275, 8556-8563

54. Gao, T., Bunemann, M., Gerhardstein, B. L., Ma, H., and Hosey, M. M. (2000) Role of the $\mathrm{C}$ terminus of the alpha $1 \mathrm{C}(\mathrm{CaV} 1.2)$ subunit in membrane targeting of cardiac Ltype calcium channels. J Biol Chem 275, 25436-25444.

55. Striessnig, J., Ortner, N. J., and Pinggera, A. (2015) Pharmacology of L-type Calcium Channels: Novel Drugs for Old Targets? Current molecular pharmacology 8, 110-122

56. Huang, H., Ng, C. Y., Yu, D., Zhai, J., Lam, Y., and Soong, T. W. (2014) Modest CaV1.342-selective inhibition by compound 8 is beta-subunit dependent. Nat Commun 5, 4481

57. Xu, W., and Lipscombe, D. (2001) Neuronal Ca(V)1.3alpha(1) L-type channels activate at relatively hyperpolarized membrane potentials and are incompletely inhibited by dihydropyridines. The Journal of neuroscience : the official journal of the Society for Neuroscience 21, 5944-5951

58. Wei, X. Y., Perez-Reyes, E., Lacerda, A. E., Schuster, G., Brown, A. M., and Birnbaumer, L. (1991) Heterologous regulation of the cardiac Ca2+ channel alpha 1 subunit by skeletal muscle beta and gamma subunits. Implications for the structure of cardiac L-type Ca2+ channels. J.Biol.Chem. 266, 21943-21947

59. Rizzo, M. A., Springer, G. H., Granada, B., and Piston, D. W. (2004) An improved cyan fluorescent protein variant useful for FRET. Nat Biotechnol 22, 445-449

60. Nagai, T., Ibata, K., Park, E. S., Kubota, M., Mikoshiba, K., and Miyawaki, A. (2002) A variant of yellow fluorescent protein with fast and efficient maturation for cell-biological applications. Nat Biotechnol 20, 87-90 